\title{
Study on Low-velocity Impact Resistance of Aluminum Sandwich Honeycomb Panel
}

\author{
Xiao-Kang Wang ${ }^{1,2}$, Yong-Chun Yang ${ }^{1,2, *}$, Xiu-Yi Nie ${ }^{1,2}$ and Guo-Xu Niu ${ }^{1,2}$ \\ ${ }^{1}$ College of Engineering, Ocean University of China \\ Qingdao, Shandong, China \\ ${ }^{2}$ Shandong Provincial Key Laboratory of Ocean Engineering, Ocean University of China \\ Qingdao, Shandong, China \\ *Corresponding author
}

Keywords: Aluminum Honeycomb Sandwich Panel, Low-velocity Impact, Experimental Research.

\begin{abstract}
This article had briefly introduced the structural characteristics of aluminum honeycomb sandwich panel and its application in some field firstly. The Low-velocity impact resistance of aluminum honeycomb sandwich panels under variable parameters was studied. The influence of structural parameters, such as cell wall thickness, cell side length and impact panel thickness, on the low-speed impact resistance of aluminum honeycomb sandwich panels was summarized which can provide some guidances for the design of honeycomb panels with reasonable structural parameters.
\end{abstract}

\section{Introduction}

The honeycomb panel has the advantages of light weight, high specific strength, high specific stiffness, impact resistance, sound insulation, heat insulation and shock absorption [1], since the fifties of the 20th century, which is widely used in the fields of aerospace, automotive, shipbuilding, construction and other fields [2]. Honeycomb panel is a layered composite structure material, which consists of two thin and high-strength panels and a layer of thick and very light core material through a combination of certain technology. The panel is mostly made of steel, aluminum, stainless steel or alloys, etc. [3]. The core is metal honeycomb core, foam (resin) core material or a corrugated core material and so on and it acts as a connecting and support panels in a honeycomb structure. Shapes of the honeycomb core can generally be divided into hexagonal, triangular, five-pointed star-shaped, square, diamond, etc [4].

For aluminum honeycomb sandwich structures, various low-speed impact events are often unavoidable during the periods of production, maintenance and service. Typical impact events include equipment collision, tool drop, etc. [5] and some data indicated that the low-speed impact of the damage could lead to that aluminum honeycomb sandwich composite surface compression strength reduced by more than half [6]. It has great significance for the aluminum honeycomb core material on the outer surface of the mechanical properties under the Impact of low-energy for sake of security. The impact problem of the honeycomb panel is a complex nonlinear transient response, and it is difficult to study the problem in a theoretical and analytical way. In recent years, domestic scholars often use finite element simulation to study the impact of honeycomb plate problem, but few people carry out in-depth experimental study of the problem. In this paper, a large number of free-falling ball impact tests were carried out on aluminum honeycomb sandwich panels with different structural parameters. The influence of the structural parameters of the aluminum honeycomb sandwich panel on the depth of the impact dent was summarized, which will provide some guidance for the subsequent design of the honeycomb panel with reasonable structural parameters. 


\section{Low-velocity Impact test of Aluminum Honeycomb Sandwich Panel}

\section{Materials and Samples}

For convenience of description, this paper defined the upper panel as the impact panel, the lower panel as the non-impact panel. The material of upper and lower panel are the aluminum alloy 5052 and the honeycomb core is the aluminum alloy 3003. The total specimen geometry is $220(\mathrm{~L}) * 160(\mathrm{w}) * 22 \mathrm{~mm}(\mathrm{t})$. We selected 12 kinds of samples with different structural parameters in the following impact tests and theirs specific parameters are shown in Table 1.

\section{Experimental Test Equipments and Methods}

The impact test was carried out by using a falling ball tester. The specimens were fixed on the steel base of the testing machine, and the impact ball with diameter of $50 \mathrm{~mm}$ was taken. The height between the steel plate and the upper panel of the honeycomb panel was $2000 \mathrm{~mm}$. To prevent the mutual interference between the impact points, the distance between the impact points and between the impact point and edges of the specimen were all greater than $50 \mathrm{~mm}$. So that each sample had four to six (the number depends on the size of the injury area) adjacent impact dents, as is shown in Figure 1.

Table 1. Test sample specification ( $\mathrm{mm})$

\begin{tabular}{|c|c|c|c|c|}
\hline Numbering & $\begin{array}{l}\text { Lower panel thickness } \\
t_{d}\end{array}$ & $\begin{array}{l}\text { Upper panel thickness } \\
t_{u}\end{array}$ & $\begin{array}{l}\text { Cell side length } \\
L_{\mathrm{s}}\end{array}$ & $\begin{array}{l}\text { Cell wall thickness } \\
t_{w}\end{array}$ \\
\hline $1-1$ & 1 & 0.5 & 4 & 0.035 \\
\hline $1-2$ & 1 & 0.5 & 4 & 0.05 \\
\hline $1-3$ & 1 & 0.5 & 4 & 0.065 \\
\hline $1-4$ & 1 & 0.5 & 4 & 0.08 \\
\hline $2-1$ & 1 & 0.5 & 3 & 0.035 \\
\hline $2-2$ & 1 & 0.5 & 4 & 0.035 \\
\hline $2-3$ & 1 & 0.5 & 5 & 0.035 \\
\hline $2-4$ & 1 & 0.5 & 6 & 0.035 \\
\hline $3-1$ & 1 & 0.5 & 3.7 & 0.065 \\
\hline $3-2$ & 1 & 1 & 3.7 & 0.065 \\
\hline $3-3$ & 1 & 1.5 & 3.7 & 0.065 \\
\hline $3-4$ & 1 & 2 & 3.7 & 0.065 \\
\hline
\end{tabular}

Note: The upper panel in Table 1 is the impact panel.

The depth of dent after impact was more likely to reflect the damage trend of honeycomb structure composites than damage area $[7,8]$. Therefore, the depth of dent was used to characterize the damage characteristics by low-velocity impact for the aluminum honeycomb sandwich panel. After the impact test, we used laser displacement sensors and data acquisition instrument to measure the depth of dents, as is shown in Figure 2. The range of displacement sensor range is $8 \mathrm{~mm}$ and its precision is $0.001 \mathrm{~mm}$. 


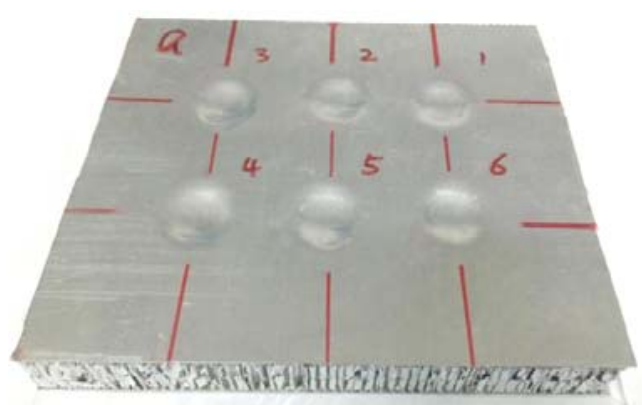

Fig. 1.The sample after impact

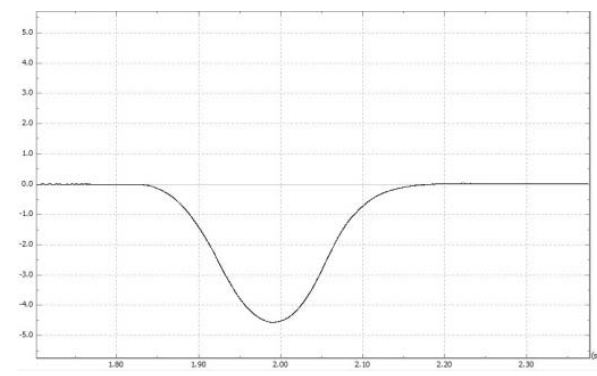

Fig. 2.The laser scanning of dent

\section{Experimental Test Results and Analysis}

The paper presented an influence of different structural parameters (upper panel thickness, cell wall thickness, cell side length) on the impact resistance of aluminum honeycomb sandwich panels under the same low-velocity impact energy. Only the depth of indent formed after the impact was considered in the test and the effects of different structural parameters on energy absorption are discussed in the subsequent simulations.

\section{Effect of Cell Density on the Impact Results}

The cell density $[9,10]$ is calculated using the formula below:

$$
\rho_{\mathrm{ca}}=\frac{8 \mathrm{~d} \cdot t_{w}}{A} \cdot \rho_{c} \approx \frac{8}{3 \sqrt{3}} \frac{t_{w}}{\mathrm{~L}_{\mathrm{s}}} \rho_{c}
$$

Where $\rho_{c}$ is the material density of cell, $t_{w}$ is the wall thickness of cell and $L_{\mathrm{s}}$ is the side length of cell. As is shown in Table 1, eight cell densities of aluminum honeycomb sandwich panels were selected by varying cell wall thickness and cell length and the effect of single parameter on cell impact resistance was studied, which was based on the variable-controlling approach. The cell wall thickness ${ }^{t_{w}}$ were taken as $0.035 \mathrm{~mm}, 0.05 \mathrm{~mm}, 0.065 \mathrm{~mm}, 0.08 \mathrm{~mm}$ respectively and cell side lengths $L_{\mathrm{s}}$ were taken as $3 \mathrm{~mm}, 4 \mathrm{~mm}, 5 \mathrm{~mm}$ and $6 \mathrm{~mm}$ respectively. The search and analysis of the test results of the above test samples were shown in Table2.

Table 2. Depth of dent corresponding to different cell densities

\begin{tabular}{|l|l|l|l|l|l|l|l|l|}
\hline Numbering & $1-1$ & $1-2$ & $1-3$ & $1-4$ & $2-1$ & $2-2$ & $2-3$ & $2-4$ \\
\hline $\begin{array}{l}\text { Depth of } \\
\text { dent }(\mathrm{mm})\end{array}$ & 3.52 & 4.41 & 4.87 & 5.23 & 4.51 & 4.05 & 3.67 & 3.37 \\
\hline
\end{tabular}

Fig. 2 is a graph showing the cell wall thickness of the honeycomb panel and the depth of dent. We can see that under the same impact conditions, the cell wall thickness is inversely related to the impact dent. Because the honeycomb plate cell element wall thickness is larger, the honeycomb element thin wall area against impact load is bigger which effectively improved the stiffness of the honeycomb panel and has a better buffering effect, then reduce the depth of the impact dent. 


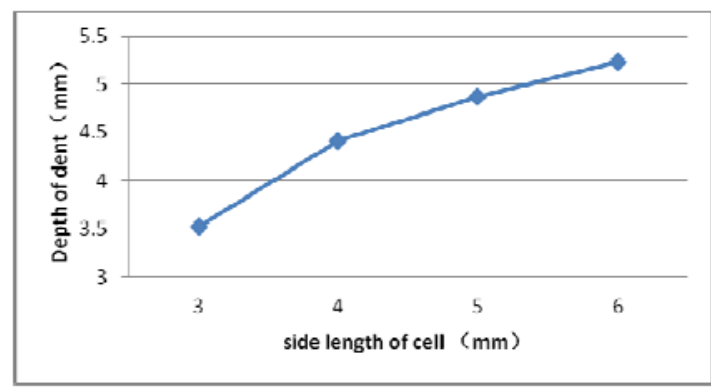

Fig. 2.Relationship between thickness of cell wall and depth of dent

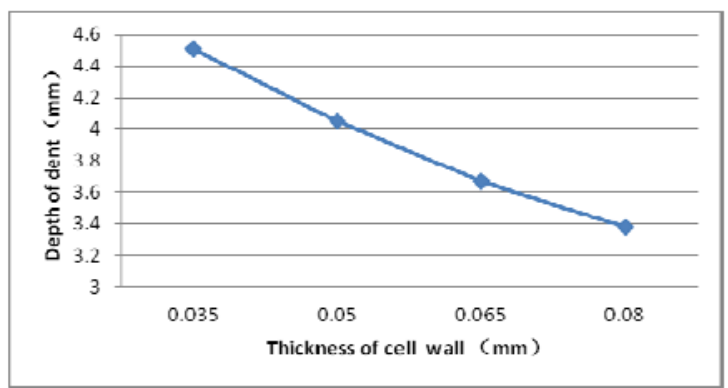

Fig. 3.Relationship between side length of cell and depth of dent

Fig. 3 is a graph showing the cell side length of the honeycomb panel and the depth of dent which we can see from that under the same conditions, the cell side length and impact dent are positively correlated. Because the honeycomb panel cell edge length increase, the number of honeycomb per unit area which withstand the impact of the load decrease accordingly. In other words, the decreased cell edge length result in a reduction in the number of thin walls of the honeycomb against the impact load, so the depth of the impact dent increase correspondingly. Increasing the cell wall thickness and decreasing the cell side length of the honeycomb panel can effectively resist the deformation of the low speed impact. In other words, increasing the cell density of the honeycomb panel can improve the performance of the honeycomb panel against impact.

\section{Influence of Thickness of Upper Panel of Honeycomb Panel on Impact}

As is shown in Table 1, four kinds of aluminum honeycomb sandwich panel samples were obtained by varying the thickness of the upper panel. The thicknesses of upper panel were taken as $0.5 \mathrm{~mm}, 1.0 \mathrm{~mm}, 1.5 \mathrm{~mm}$ and $2.0 \mathrm{~mm}$, respectively. The impact of the thickness of the upper panel on the impact resistance of the material was investigated. The results are shown in Table 3:

Table 3. Depth of the indentations for the upper panels of different thicknesses

\begin{tabular}{|c|c|c|c|c|}
\hline Numbering & $3-1$ & $3-2$ & $3-3$ & $3-4$ \\
\hline $\begin{array}{c}\text { Depth of dent } \\
(\mathrm{mm})\end{array}$ & 3.93 & 2.79 & 2.48 & 2.00 \\
\hline
\end{tabular}

Figure 4 is a plot of cell wall thickness versus depth of impact dent from which we can see that under the same impact conditions, the upper panel thickness and the impact of indentation were inversely related and increasing the thickness of the upper panel can effectively improve the impact resistance of honeycomb panels and decrease the damage by impact.

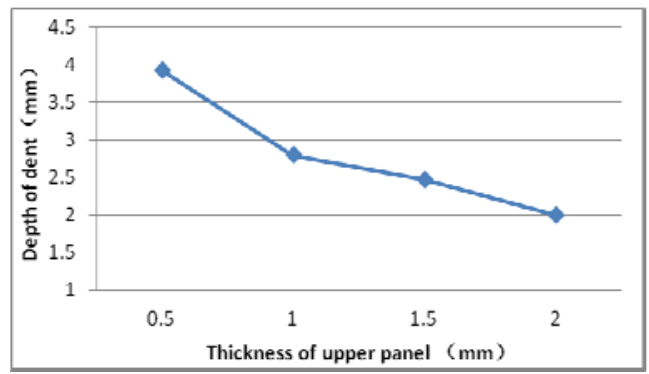

Fig.4.Relationship between thickness of upper panel and depth of dent

\section{Results and Discussions}

The analysis of the above experimental results showed that when the thickness of cell wall increased by $129 \%$, the depth of dent decreased by $25 \%$. When the cell length reduced by $50 \%$, the 
depth of dent decreased by $33 \%$. When the thickness of the upper panel increased by $300 \%$, the dent depth reduced by $49 \%$. So the depth of dents is most sensitive to the change of cell side length, followed by the cell wall thickness, and the thickness of the upper panel is the smallest and the former is much larger than the latter two, that is, the change of cell side length has the greatest effect on its low-speed impact resistance. Therefore, when we want to change the structural parameters to improve the aluminum honeycomb sandwich panels of low-speed impact performance, it is generally preferred to adjust the size of the cell side length.

\section{Conclusion}

In this paper, a series of free-falling ball impact tests were carried out on aluminum honeycomb sandwich panels with different structural parameters. The depth of the dent by impact was measured by a high accuracy laser displacement sensor and a data acquisition instrument. It is pointed out that the deformation mainly occurs in the contact collision area, and the impact panel is locally permanently collapsed at the impact site. The depth of the dent decreases from the center of the impact to the surrounding area, and there is no penetration and panel peeling.

This paper summarizes the impact of cell wall thickness, cell side length and impact panel thickness on the impact resistance of aluminum honeycomb sandwich panels, that is, reducing cell length, increasing cell wall thickness and upper panel thickness can effectively improve the impact resistance of honeycomb panels and reduce the impact damage. The change of cell side length had the greatest impact on its low-speed impact resistance. Therefore, when we want to change the structure parameters to improve the anti-low-speed performance of aluminum honeycomb sandwich panels, it is generally preferred to adjust the size of the cell side length. The results obtained in this paper can be used to validate the accuracy of subsequent simulation results and provides some guidance for the subsequent design of the honeycomb panel with more reasonable structure parameters in marine engineering.

\section{References}

[1] Tuxing Ye, Yang Yunbiao, Zeng Jingcheng, etc. Sandwich structure of composite material design principles and application [M]. Beijing Chemical Industry Press. (2007)4-8.

[2] SANDCORe. Best Practice Guide for Sandwich Structures in Marine Applications.1999.

[3] Yang Tang. Experimental Study on Failure Mode and Fatigue Performance of Honeycomb Sandwich Structures, Qingdao, The Ocean University of China, 2015.

[4] Xie Zongdai, Study on Damage Tolerance of Low Speed Impact Damage of Composite Sandwich Structure [J]. National Composites Seminar (2007)12: 21-24.

[5] Guo Zelu, Study on dynamic response characteristics of aluminum honeycomb sandwich panels under impact loading, Wuhan, Huazhong University of Science and Technology, 2014.

[6] Ren Lili, Optimal Design of Crashworthiness of Honeycomb Sandwich Plate, Changsha, Hunan University, 2012.

[7] Wang Kun. Brazing honeycomb aluminum plate quasi-static pressure and dynamic impact of numerical simulation of performance, Kunming University of Science and Technology, 2012.

[8]Meo M, Vignjevic R, Marengo G, The response of honeycomb sandwich panels under low-velocity impact loading [J]. Int J Mechan Sci( 2005)47(9):1301.

[9]Zhang Yanchang, Wang Zili. Study on crash worthiness of honeycomb sandwich panel under lateral dynamic load.[J]. J Jiangsu University of Science and Technology.21(2007)(3):1.

[10]G.R. Johnson and W.H. Cook, in: Proceedings of the Seventh International Symposium on Ballistics, The Hague, Netherlands (1983), pp. 541-547. 